\title{
Characterization of ultra-shallow aluminum implants in silicon by grazing incidence and grazing emission X-ray fluorescence spectroscopy
}

\author{
P. Hönicke, ${ }^{* a}$ Y. Kayser, ${ }^{b}$ B. Beckhoff, ${ }^{a}$ M. Müller, ${ }^{a}$ J.-Cl. Dousse, ${ }^{b}$ J. Hoszowska ${ }^{b}$ and S. H. Nowak ${ }^{b}$
}

\begin{abstract}
In this work two synchrotron radiation-based depth-sensitive X-ray fluorescence techniques, grazing incidence X-ray fluorescence (GIXRF) and grazing emission X-ray fluorescence (GEXRF), are compared and their potential for non-destructive depth-profiling applications is investigated. The depth-profiling capabilities of the two methods are illustrated for five aluminum-implanted silicon wafers all having the same implantation dose of $10^{16}$ atoms per $\mathrm{cm}^{2}$ but with different implantation energies ranging from $1 \mathrm{keV}$ up to $50 \mathrm{keV}$. The work was motivated by the ongoing downscaling effort of the microelectronics industry and the resulting need for more sensitive methods for the impurity and dopant depth-profile control. The principles of GIXRF and GEXRF, both based on the refraction of $\mathrm{X}$-rays at the sample surface to enhance the surface-to-bulk ratio of the detected fluorescence signal, are explained. The complementary experimental setups employed at the Physikalisch-Technische Bundesanstalt (PTB) for GIXRF and the University of Fribourg for GEXRF are presented in detail. In particular, for each technique it is shown how the dopant depth profile can be derived from the angular intensity dependence of the $\mathrm{Al} \mathrm{K} \alpha$ fluorescence line. The results are compared to theoretical predictions and, for two samples, crosschecked with values obtained from secondary ion mass spectroscopy (SIMS) measurements. A good agreement between the different approaches is found proving that the GIXRF and GEXRF methods can be efficiently employed to extract the dopant depth distribution of ionimplanted samples with good accuracy and over a wide range of implantation energies.
\end{abstract}

\section{Introduction}

The ongoing downsizing of the lateral component dimensions in ultra-large-scale integrated (ULSI) circuits technology requires so-called ultra-shallow junctions (USJ) with junction depths in the $20 \mathrm{~nm}$ regime and below to avoid leakage currents. Ultrashallow dopant distributions can be formed by means of ultralow energy (ULE) ion implantation with subsequent low thermal budget annealing. However, accurate measurement techniques for ultra-shallow dopant profiles are required in order to characterize and develop ULE implantation and rapid thermal annealing (RTA) processes.

Secondary ion mass spectrometry (SIMS) is a well-established depth-profiling technique and numerous efforts have been made to apply SIMS to USJ characterization. However, due to various effects, especially in the first few nanometers, SIMS leads to increased uncertainties in the profile shape as well as in the determined doses. ${ }^{1}$ Several different approaches to improve or overcome these difficulties have been adopted and are reported in the literature. ${ }^{2-4}$ In addition, the research on complementary

${ }^{a}$ Physikalisch-Technische Bundesanstalt, Abbestr. 2-12, 10587 Berlin, Germany. E-mail: philipp.hoenicke@ptb.de

${ }^{b}$ Department of Physics, University of Fribourg, Chemin du Musée 3, CH1700 Fribourg, Switzerland techniques for USJ characterization has been significantly intensified. ${ }^{5-7}$ Depth-profiling methods based on electrons suffer from the short mean penetration depth of the electrons and related quantification challenges while ion scattering techniques do not usually provide a sufficient depth resolution for characterizing USJ. In this paper it is shown that grazing incidence and emission X-ray fluorescence methods allow non-destructive, quantitative depth-profiling of implanted dopants with a depth resolution in the low nanometer range.

\section{GIXRF and GEXRF}

To date, for the characterization of ultra-shallow implants by means of X-ray fluorescence (XRF), grazing incidence (GIXRF) in the hard X-ray range has mainly been employed. ${ }^{5,8}$ In grazing incidence XRF, the incident angle between the sample surface and the incoming monochromatic X-ray beam is varied around the critical angle of total external reflection. This configuration takes advantage of the angle and photon energy dependent X-ray standing wave (XSW) field that originates from the interference between the incoming and reflected beams. The wave field intensity distribution is changed when varying the incidence angle. These changes are used in GIXRF in order to characterize periodic multilayers, ${ }^{9}$ adsorbed molecules ${ }^{10}$ and thin layers. ${ }^{11}$ The 
penetration depth depends on the incident angle and is usually in the $\mathrm{nm}$ range. This results in a rather low scattering background.

The Physikalisch-Technische Bundesanstalt (PTB), Germany's national metrology institute, developed a GIXRF approach using soft X-ray radiation, enabling accurate depth profiling as well as dose quantification of various implanted mid- $Z$ and even light elements down to boron. This reference-free ${ }^{12}$ approach is based on ab initio calculations, using the simulated XSW field, relevant atomic fundamental parameters and an assumed dopant profile which is varied to fit the experimental data. This method does not rely on any calibration samples or subsequent etching of the sample and is described in detail in ref. 13.

Like GIXRF, the grazing emission X-ray fluorescence (GEXRF) technique can also be used to determine elemental depth profiles of ULE implants. ${ }^{14,15}$ In GEXRF the fluorescence signal is observed relative to the surface under different shallow emission angles in the vicinity of the critical angle. Because of the refraction of the fluorescence X-rays at the surface and the large effective path lengths for the fluorescence X-rays, the probed depth region is limited to a scale ranging from a few $\mathrm{nm}$ to several hundred $\mathrm{nm}$, depending on the emission angle. The extinction depths of GIXRF and GEXRF are thus comparable. However, due to the large effective path lengths, GEXRF should be more sensitive to the sample matrix compared to GIXRF. This is due to the lower photon energy of the fluorescence X-rays compared to the incident X-rays. Indeed, the experimental differences between the two geometries are given by the different critical angles and matrix absorption coefficients for the X-ray wavelengths of interest (incident X-rays or fluorescence X-rays). GEXRF is used for similar applications, i.e., the analysis of thin layers ${ }^{16,17}$ or particles deposited on a surface. ${ }^{18}$ The oscillations observed in the angular intensity behavior in GEXRF are due to interferences between the fluorescence X-rays following different detection paths while in GIXRF, the oscillations originate from the intensity changes of the XSW field distribution.

At the University of Fribourg, a grazing emission setup based on the high-resolution von Hamos curved crystal X-ray spectrometer $^{19}$ was developed. This combination is a natural one because the collimation of the fluorescence X-rays required by the grazing emission geometry to define the emission angle is automatically realized by the Bragg diffraction crystal in the von Hamos geometry. In the latter geometry, the grazing emission conditions are realized by turning the target surface close to the emission direction defined by the Bragg angle which depends on the lattice spacing of the diffraction crystal and the measured fluorescence wavelength. The angular resolution of the Fribourg von Hamos spectrometer is sufficient to realize grazing emission experiments without any refinement of the resolution by slits.

GEXRF was first introduced by Becker et $a .^{20}$ as an equivalent geometry to GIXRF according to the principle of microscopic reversibility. In GIXRF the excitation of the fluorescence radiation is restricted towards a surface-near region, whereas in GEXRF the detection of the fluorescence X-rays is confined to those emitted from a surface-near region. The performances of both grazing XRF techniques have previously been compared on a theoretical basis to evaluate the detection limits ${ }^{21}$ and on an experimental basis by using a plate beamguide ${ }^{22}$ or by studying arsenic traces on wafer surfaces. ${ }^{23}$ The general conclusion from these studies is that, despite the better signal-to-background ratios offered by wavelength-dispersive GEXRF setups, GIXRF provides better detection limits because of the higher luminosity of grazing incidence setups. For Al surface contaminations on silicon, detection limits of $3.7 \times 10^{12} \mathrm{~cm}^{-2}$ for GEXRF ${ }^{24}$ and $2 \times 10^{9} \mathrm{~cm}^{-2}$ for total-reflection $\mathrm{XRF}^{25}$ (TXRF) were reported. When going from surface contaminations to depth profiling and from TXRF to GIXRF, the TXRF detection limits degrade with the rising incident angle and the increasing mean penetration depth due to the higher spectral background contributions when exciting above the critical angle. On the other hand, GEXRF setups are more suitable for lateral surface mapping applications since collimated or micro-focused beams can be easily implemented. For GEXRF the lateral resolution is given by the beam size and the orientation of the sample with respect to the beam, because of the shallow observation angles the fluorescence source appears to be line-like to the detection setup. The lateral analysis area in GIXRF is rather large due to the shallow incidence angles between the sample surface and the X-ray beam, resulting in an extension of the footprint in one dimension. Especially in the low angle regime, the lateral analysis area is therefore defined by the solid angle of detection and its angle dependent determination becomes crucial. ${ }^{25}$ In general both techniques can be used for non-destructive surface or nearsurface analysis purposes in terms of elemental and structural composition. If needed, they can also be combined with diffraction $^{26,27}$ or absorption ${ }^{28,29}$ techniques to study, for example, nearest-neighbor configurations.

In this work, the PTB GIXRF method ${ }^{13}$ is compared to the GEXRF approach of the University of Fribourg ${ }^{14}$ for the characterization of ultra-shallow aluminum implants in silicon with various implantation energies. Additionally, TRIM calculations $^{30}$ as well as SIMS measurements on selected samples are used to gain further information about the reliability of the XRF results obtained in the respective grazing geometries.

\section{Experimental and data analysis}

Five different Al-implanted silicon wafers were characterized by means of GIXRF and GEXRF. All samples were implanted at a dose of $10^{16}$ atoms per $\mathrm{cm}^{2}$ and the respective implantation energies were $1 \mathrm{keV}, 5 \mathrm{keV}, 10 \mathrm{keV}, 15 \mathrm{keV}$ and $50 \mathrm{keV}$.

The GIXRF measurements were conducted at the plane grating monochromator (PGM) beamline for undulator radiation at BESSY, ${ }^{31}$ where monochromatized undulator radiation in the photon energy range of $78 \mathrm{eV}$ to $1860 \mathrm{eV}$ is available. The beam size in the focus plane of the PGM beamline is about $140 \mu \mathrm{m}$ in the horizontal and $20 \mu \mathrm{m}$ in the vertical direction. The latter can be varied in line with the exit slit size. At an incident angle of $0.9^{\circ}$ this corresponds to a footprint size of $9 \mathrm{~mm}$ by $20 \mu \mathrm{m}$. The measurements were carried out using PTB's radiometrically calibrated instrumentation ${ }^{12}$ under high vacuum conditions. This includes a known solid angle of detection ${ }^{25}$ (the distance between the sample and the detector was about $23 \mathrm{~mm}$ ), calibrated photodiodes to determine the incident photon flux and a calibrated silicon drift detector (SDD) with known spectral response functions and efficiency. In combination with the use of tabulated or measured ${ }^{32}$ fundamental parameters for the relevant atomic processes, e.g., the photo-ionization cross-section and the 
fluorescence yield of the elements of interest, a fully referencefree quantification approach was applied. ${ }^{13}$

The fluorescence spectra were recorded in steps of $0.05^{\circ}$ over an incident angular range spanning from $0^{\circ}$ to $4.25^{\circ}$. The incidence angle was defined relative to the sample surface and the incident X-ray beam at an energy of $1622 \mathrm{eV}$. The SDD spectra were then deconvoluted using spectral response functions and both the Bremsstrahlung and the Resonant Raman Scattering (RRS) backgrounds originating from the silicon substrate ${ }^{33}$ to derive the incident-angle dependent fluorescence intensity of the $\mathrm{Al} \mathrm{K} \alpha$ fluorescence radiation. This intensity was used to determine the implanted depth profile by a straightforward calculation. The routine, described in detail in ref. 13, uses an incidentangle dependent convolution of the $\mathrm{IMD}^{34}$ calculation of the XSW field, a mathematical model of the depth profile as well as a correction term for the absorption of the excited fluorescence radiation. All relevant geometrical and experimental parameters, i.e., the solid angle of detection or the incident photon flux, are included to gain quantitative information about the depth profile. An analytical function describing the shape, position and area of the depth profile is used and the fitting parameters are varied until the best agreement between the measurement and the model is obtained in the fitting procedure. For the Al implanted $\mathrm{Si}$ samples considered in this work, an asymmetric Gaussian function was found to be the most suitable one to describe the results of the TRIM calculations. ${ }^{30}$ A constant parameter relation between the center position of the depth distribution and the implantation energy was derived from TRIM to reduce the amount of free fitting parameters and thus to improve the convergence of the iterative procedure related to the forward calculation scheme.

The GEXRF measurements were performed at the European Synchrotron Radiation Facility (ESRF) ID21 beamline with the Fribourg von Hamos spectrometer ${ }^{19}$ under vacuum conditions. The latter was equipped with an $\operatorname{ADP}(101) \operatorname{crystal}(2 d=10.642 \AA$, curvature radius $=25.4 \mathrm{~cm})$ and a back-illuminated CCD $(1340 \times$ 400 pixels of $20 \times 20 \mu \mathrm{m}^{2}$ ) for the X-ray detection. The covered energy range, limited by the horizontal extension of the CCD, was about $40 \mathrm{eV}$ for the presented measurements. During the acquisition of an angular profile the crystal and detector positions were kept fixed, and thus the solid angle is constant. The synchrotron radiation beam was produced by a wiggler. Higher order harmonics rejection and monochromatization were realized by means of $\mathrm{Si}$ mirrors and $\mathrm{Ni} / \mathrm{B}_{4} \mathrm{C}$ multilayers. The $\mathrm{Si}$ and $\mathrm{Al} \mathrm{K \alpha}$ fluorescence was excited at $2000 \mathrm{eV}$ and $1582 \mathrm{eV}$ with a beam of about $1 \mathrm{~mm}$ in diameter. The choice of the latter excitation energy was guided by the photon energy dependence of the edge position of the Si RRS involving the K- and L-shells. ${ }^{35}$ Thanks to the highresolution of the von Hamos spectrometer and the careful optimization of the experimental fluorescence excitation conditions, the X-ray signals originating from the $\mathrm{Al} \mathrm{K} \alpha$ fluorescence line and the Raman scattering from the bulk $\mathrm{Si}$ could be completely separated. ${ }^{14}$ Thus, no deconvolution of the acquired spectra was necessary. Due to the clean background conditions, the raw count rate in the acquired $\mathrm{Al} \mathrm{K} \alpha$ spectra was considered in the angular intensity profiles. However, in contrast to the GIXRF measurements, the angular profiles of the two fluorescence lines of interest had to be recorded sequentially. For each sample, the angular intensity profile of the $\mathrm{Al} \mathrm{K} \alpha$ line was recorded for 100 seconds at
100 different emission angles each separated by $0.0225^{\circ}$, whereas the intensity of the $\mathrm{Si} \mathrm{K} \alpha$ line was measured at 40 different points separated by $0.0563^{\circ}$ with a collecting time of 20 seconds per point. The incident angle between the sample surface and the exciting X-ray beam, which is defined for each sample position with respect to the Bragg angle of the fluorescence line, increased with the grazing emission angle and varied from $38.4^{\circ}$ to $40.6^{\circ}$ and $48.0^{\circ}$ to $50.2^{\circ}$ in the measurements of the $\mathrm{Al}$ and $\mathrm{Si} \mathrm{K} \alpha$ angular profiles.

The angular intensity profiles of the Al dopants were fitted by means of the equation quoted in ref. 36 for ion-implanted samples. The continuous depth-dependent dopant density function was assumed to correspond, like in the analysis of the GIXRF measurements, to a joined half-Gaussian distribution in order to account for asymmetric dopant profiles. The common center and the standard deviations of the two half-Gaussians were the independent free fitting parameters. This approach to fit the angular intensity profile with a known analytical function describing the implantation profile was already successfully applied in ref. 14. In the equation mentioned above, besides the dopant distribution, the surface transmission factor (incorporating the change in the field strength) at the sample-vacuum interface for the fluorescence radiation and the pronounced absorption due to the large effective path length within the sample are the main factors modeling the angular dependence of the fluorescence intensity. In GIXRF, to model the angular dependence, one needs to account for the creation of an XSW field (because of the reflection at the vacuum-sample interface for incidence angles below the critical angle) and an increased absorption in the depth direction due to the shallow incidence angles. In both geometries the refraction of X-rays at the sample surface and the momentum and energy conservation imply that an evanescent wave propagating along the sample surface has to be considered, the detected fluorescence intensity for angles below the critical angle originating from this near-interface region. ${ }^{20,37}$

Exact knowledge of the absolute angular scale is essential for both grazing XRF techniques in order to derive accurate depth profiles from the angular behavior of the fluorescence intensity. The instrumental angular positions of the two setups were therefore calibrated using the derivative of the angular intensity curve of the Si fluorescence lines. The offset of the instrumental angular axis for each sample was determined relative to the respective critical angle by comparing the results of a Gaussian fit of the derivative of the measured bulk fluorescence curve and the calculated reflectivity curve of plain silicon ${ }^{38}$ for the respective photon energy. The validity of this approach was checked for each sample by means of X-Ray Reflectometry (XRR) measurements in the $\theta-2 \theta$ mode at $1622 \mathrm{eV}$ and $1740 \mathrm{eV}$, the $\mathrm{X}$-ray energies of interest for the calibration of the GIXRF and GEXRF setups. The left-hand side of Fig. 1 shows a comparison between the corrected angular GEXRF curve for the Si-K $\alpha$ fluorescence radiation, the measured XRR signal at $1740 \mathrm{eV}$ and the calculated reflectivity for plain silicon at $1740 \mathrm{eV}$. The measured XRR curve below $0.9^{\circ}$ is lower than the theoretical one because the footprint of the beam at very shallow angles is larger than the sample. Thus, the incident beam cannot be fully reflected. The right-hand side of Fig. 1 shows the respective derivatives, which were partially scaled and normalized for better 

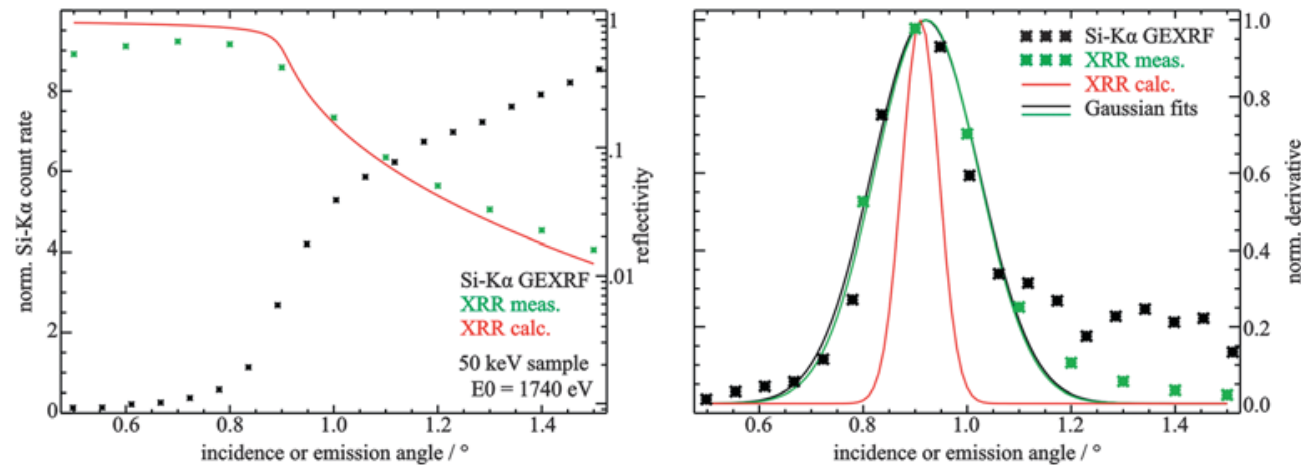

Fig. 1 Comparison of the measured reflectivity (XRR) and silicon fluorescence intensity measured in GEXRF to the calculated XRR curve (left-hand side) and the derived critical angles for the $50 \mathrm{keV}$ implant sample (right-hand side). The critical angle corresponds to the inflection point. The latter is given for each curve by the center of a Gaussian fit to the normalized first derivative (dots, right-hand side).

comparability, as well as Gaussian fits to the derivative of the GEXRF curve and the experimental XRR curve. The center positions of the Gaussians, which correspond to the critical angle, are in good overall agreement. ${ }^{38}$ This behavior was found for all samples and proves that the critical angle of the bulk silicon fluorescence lines is not influenced by the $\mathrm{Al}$ ion implantation despite the high dose.

The SIMS measurements for the $15 \mathrm{keV}$ and the $50 \mathrm{keV}$ samples were performed by RTG Mikroanalyse GmbH. For comparison to the GIXRF and GEXRF results, a Cameca ims $4 f$ instrument with $8 \mathrm{keV} \mathrm{O}_{2}{ }^{+}$was used to obtain qualitative depth profiles for these high energy implants. The depth scale of the SIMS measurements was calibrated by measuring the final crater depth.

\section{Results and discussion}

The depth profiles determined by GIXRF and GEXRF for the $\mathrm{Al}$ implantations realized at $1 \mathrm{keV}, 5 \mathrm{keV}$ and $10 \mathrm{keV}$, respectively, are shown in Fig. 2. For comparison, the calculated TRIM $^{30}$ depth profiles are also included. In contrast to the TRIM calculations and the profiles obtained by GEXRF, the GIXRF method returns depth profiles that are shifted towards the surface while the fitted profile widths are similar to the ones

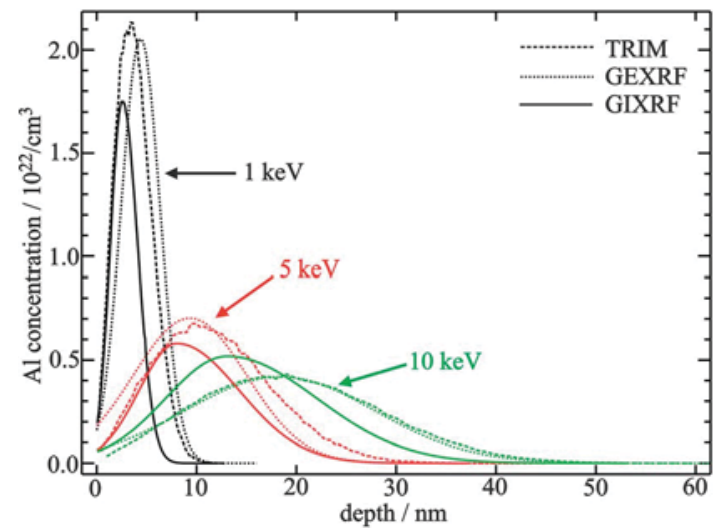

Fig. 2 The determined depth profiles for the $1 \mathrm{keV}$ (black), the $5 \mathrm{keV}$ (red) and the $10 \mathrm{keV}$ (green) $\mathrm{Al}$ implantations in Si. The GIXRF (solid lines) and GEXRF ${ }^{14}$ (dotted lines) results are shown and compared to TRIM calculations ${ }^{30}$ (dashed lines). retrieved with TRIM and GEXRF. The GEXRF depth profile curves agree very well with the TRIM calculations regarding the projected range and the depth-profile width. The small variations in the maximum positions of the profile cannot be explained by a native oxide layer on top of the $\mathrm{Si}$. The thickness of the oxide was quantified by XRF for each sample (see Table 1). An inclusion of the oxide in the TRIM calculation for the considered samples does not alter the shape or the position of the returned dopant depth distribution. Neither does the calculated GEXRF angular intensity curve reveal any significant differences despite the slightly different refractive index of $\mathrm{SiO}_{2}$ with respect to $\mathrm{Si}$ for the energy of the $\mathrm{Al} \mathrm{K} \alpha$ line. The shift towards the surface of the GIXRF curves is thus an artifact of the method.

The results for the $15 \mathrm{keV}$ and the $50 \mathrm{keV}$ implantation samples (Fig. 3 and 4) support the conclusion above since the overall behavior is the same: the GIXRF depth profiles are shifted towards the surface while the returned widths of the depth profiles are similar for all three methods. Again the GEXRF results agree well with the TRIM calculations. However, the qualitative SIMS depth profile of the $15 \mathrm{keV}$ sample is also shifted towards the surface and stretches over a larger depth region compared to the results of TRIM, GIXRF and GEXRF. Due to the very high local concentration of $\mathrm{Al}$ and the shallow distribution, this is probably caused by matrix effects influencing

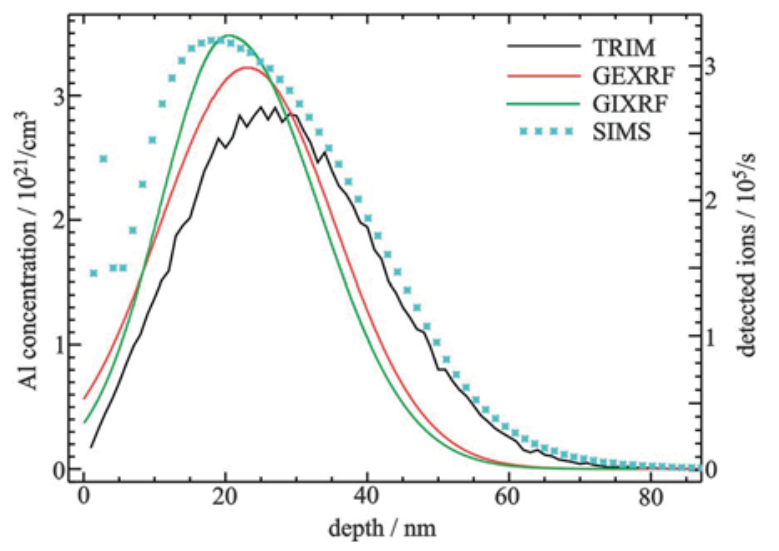

Fig. 3 Comparison of the GIXRF determined depth profile for the $15 \mathrm{keV}$ Al implantation with the GEXRF result, ${ }^{14}$ the scaled qualitative SIMS measurement (right hand $y$-axis) and the TRIM calculation. ${ }^{30}$ 


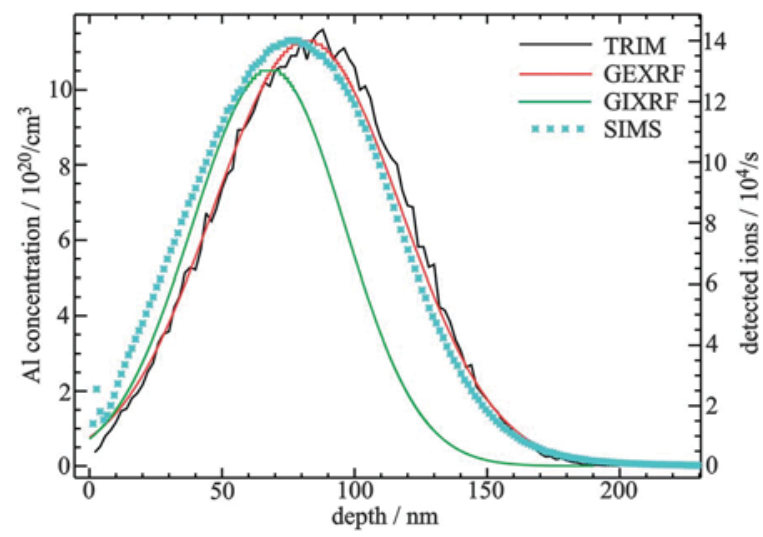

Fig. 4 Comparison of the GEXRF ${ }^{14}$ determined depth profile for the $50 \mathrm{keV} \mathrm{Al} \mathrm{implantation} \mathrm{with} \mathrm{the} \mathrm{GIXRF} \mathrm{result,} \mathrm{the} \mathrm{SIMS} \mathrm{measurement}$ and the TRIM calculation. ${ }^{30}$

both the sputtering rate and the ion yields. This leads to distortions in both the depth and the intensity axis of the SIMS measurement and illustrates thus the limits of this depth-profiling technique for very shallow near-surface implantation profiles with such a high dose.

For the sample with an implantation energy of $50 \mathrm{keV}$ (Fig. 4), for which the local $\mathrm{Al}$ concentrations are lower since the distribution extends over a larger depth region, the agreement between SIMS, GEXRF and TRIM is very good. The peak positions are slightly shifted with respect to each other but the profile widths are in principle alike. Only the depth profile determined by GIXRF presents a different behavior concerning both position and width.

The deviations between the depth profiles retrieved with GIXRF and the other approaches, essentially for the implants with the lowest energies and because of the high implantation doses, are to some extent due to the neglect of the implantation profile in the calculation of the XSW. The differences in the results suggest that the implantation profiles as well as the damage introduced to the crystal structure should significantly influence the XSW. These deviations are even enhanced by the fact that the photon energy for the GIXRF measurements was tuned to $1622 \mathrm{eV}$ to optimize the excitation conditions for the Al$\mathrm{K} \alpha$ fluorescence. The chosen energy is between the absorption edges of $\mathrm{Al}(1559 \mathrm{eV})$ and $\mathrm{Si}(1839 \mathrm{eV})$, which leads to large differences in the mass absorption cross-sections and the imaginary part $(k)$ of the refractive index for both materials. Consequently, the penetration depth and therefore the XSW are influenced. In GEXRF the energies of interest in the measurement of the angular profiles are the ones of the Si K $\alpha$ and $\mathrm{Al} \mathrm{K} \alpha$ lines. Since the latter energy is below the K-absorption edges of both elements, the difference in the optical constants of $\mathrm{Al}$ and $\mathrm{Si}$ is less pronounced. Regarding the angular intensity curves of the Si fluorescence lines with respect to which the angular scales were calibrated in GIXRF and GEXRF, the difference in the imaginary part of the refractive index is also high. The influence on the real part $(n)$ on the other hand is negligible, whereby the normalization using the critical angle of plain silicon is still valid, as confirmed by the XRR measurements.

To visualize the influence of the high local $\mathrm{Al}$ concentration for the $1 \mathrm{keV}$ sample on the real and imaginary parts of the refractive index or the optical constants, respectively, and thus the calculated XSW field, a comparison between a measured X-ray reflectivity curve and curves calculated with and without taking the implantation profile into account is shown in Fig. 5. It is obvious that there is a significant discrepancy between the measured curve and the simulation where $4.8 \mathrm{~nm} \mathrm{SiO}_{2}$ on $\mathrm{Si}$ and no $\mathrm{Al}$ profile are assumed. The measured XRR curve was then fitted using $\mathrm{IMD}^{34}$ and a layer system consisting of $\mathrm{SiO}_{2}$ on $\mathrm{Al}_{x} \mathrm{Si}_{y}$ on $\mathrm{Si}$. To take into account the implantation and the resulting amorphization, surface and interface roughness, the densities and thicknesses of $\mathrm{SiO}_{2}$ and $\mathrm{Al}_{x} \mathrm{Si}_{y}$ were used as free fitting parameters. Additionally, the optical constants ( $n$ and $k$ ) of the underlying substrate were modified accordingly. The solid XRR curve which best fits the experimental data corresponds to a $0.3 \mathrm{~nm} \mathrm{SiO}$ layer (density: $1.8 \mathrm{~g} \mathrm{~cm}^{-3}$ ) on top of a $4.1 \mathrm{~nm}$ layer of $\mathrm{Al}_{x} \mathrm{Si}_{y}$ (density: $2.9 \mathrm{~g} \mathrm{~cm}^{-3}$ ) on top of bulk silicon. Using this layer configuration, a new XSW was calculated and used in the fitting procedure to obtain the dopant depth profile. The result is shown in the left panel of Fig. 6 where the TRIM and the GEXRF results are scaled to the total retained dose determined by GIXRF. As shown, by introducing this improved XSW, the difference in the projected range between the different methods is significantly reduced.

However, this approach cannot be easily applied to the high energy implants due to the larger depth region which is affected by the implantation process. The surface and local dopant concentration levels and thus the impact on the optical constants are lower. To improve the wave field calculation for the $50 \mathrm{keV}$ sample, a different approach has to be used. In this approach based on the formalism of Klockenkämper, ${ }^{39}$ the wave field is recalculated after having applied the depth profile fitting algorithm described above. Employing the experimental XRR curve, the intensity of the XSW field at the vacuum-sample interface is evaluated. The depth-dependent decay of the intensity, which is a function of the penetration depth, is computed after each iteration for the fitted depth-profile. The fitted depth variations of the concentration values for $\mathrm{Al}$ and $\mathrm{Si}$ are estimated to deduce the effective depth-dependent values for $n$ and $k$ by means of a linear combination. These values are then used for the

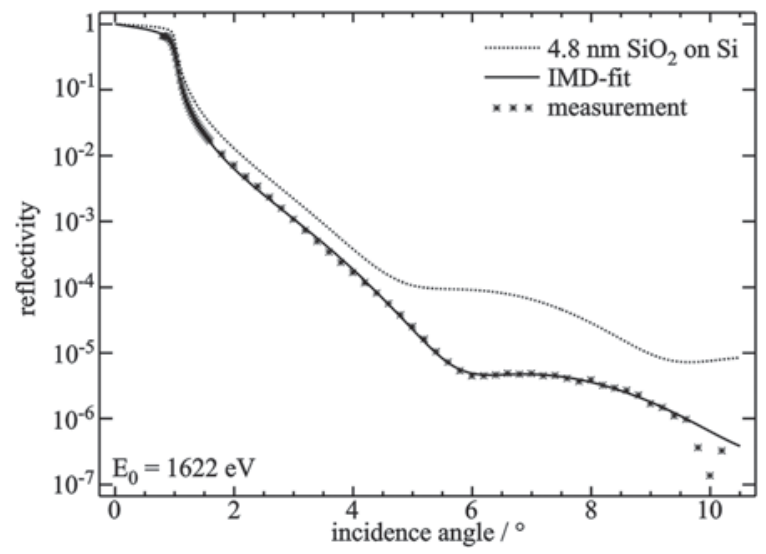

Fig. 5 Comparison between the measured reflectivity for the $1 \mathrm{keV}$ sample and an IMD calculation, ${ }^{34}$ assuming $4.8 \mathrm{~nm} \mathrm{SiO}{ }_{2}$ on $\mathrm{Si}$ (dotted line) without aluminum. The solid line corresponds to the best fit using the three-layer model described in the text. 

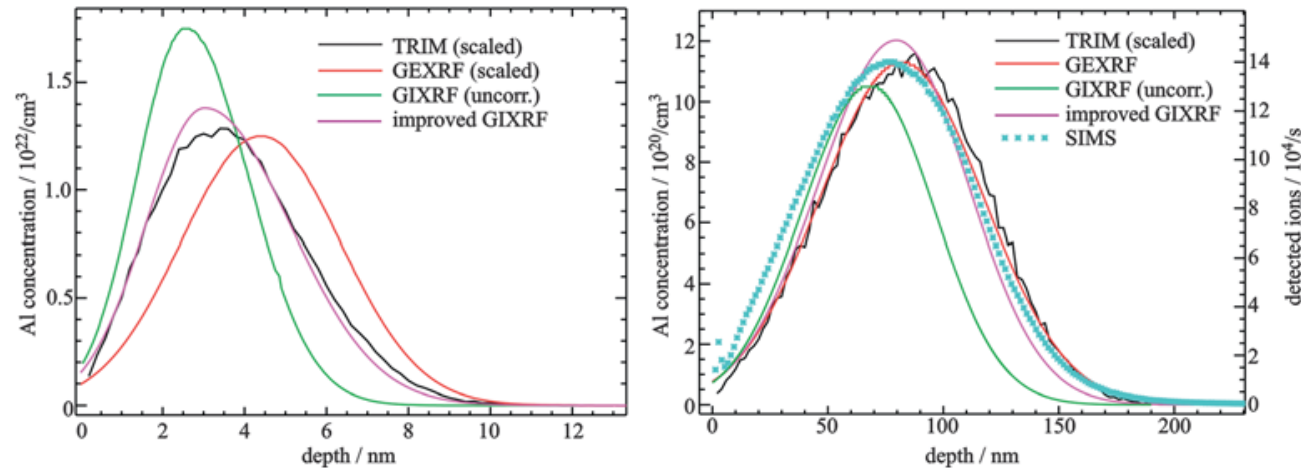

Fig. 6 Depth profiles for the $1 \mathrm{keV}$ sample (left) and the $50 \mathrm{keV}$ sample (right) determined with the improved XSW calculations taking into account the $\mathrm{Al}$ implantation (see text) in comparison to the depth profiles determined by other methods.

calculation of the effective penetration depth. This iterative approach, the recalculation of the XSW field and fitting, is repeated until the results of the fit converge to the final value.

The depth profile for the $50 \mathrm{keV}$ implant obtained with this iterative procedure is shown in Fig. 6 (right-hand side). Compared to the profile determined with the undisturbed XSW calculation (using $1.8 \mathrm{~nm} \mathrm{SiO}_{2}$ on plain $\mathrm{Si}$ ), the returned profile using the improved XSW is now in considerably better agreement with the profiles obtained with the other methods. The projected range is congruent to the ranges obtained with SIMS, TRIM and GEXRF but the overall shape retrieved with GIXRF is still somewhat narrower. The $5 \mathrm{keV}$ and the $10 \mathrm{keV}$ samples have not been reanalyzed due to limited beamtime.

Besides the shape and position of the depth profile, the total number of implanted ions can also be studied. The total retained doses determined by GIXRF for the different implanted samples are shown in Table 1 . The relative uncertainty is estimated to be $10 \%$. The main contributions to the uncertainty budget emanate from the XSW calculation and the atomic fundamental parameters used in the quantification. ${ }^{12}$ The first column shows the values determined using the undisturbed XSW calculation. The values derived from the fitting approach with the iteratively improved XSW are in the center column and the right column shows the values derived from the GEXRF measurements. The doses determined by GEXRF are not absolute numbers but were calculated with respect to a reference value. The dose determined by GIXRF for the $10 \mathrm{keV}$ sample is used here as a reference value for GEXRF. The doses for the other four samples were obtained consecutively with respect to this reference by considering the fluorescence intensities at emission angles well above the critical angle and by correcting for the self-absorption in the sample. The larger relative errors of the values obtained by GEXRF result from the normalization with the GIXRF reference value.

The $1 \mathrm{keV}$ implant shows the largest deviation to the nominal dose of $10^{16} \mathrm{~cm}^{-2}$ while the doses determined for the $10 \mathrm{keV}$ and the $15 \mathrm{keV}$ implants are in good agreement with the nominal values. The determined dose for the $50 \mathrm{keV}$ sample is also quite different from the nominal value, but the depth profile is obviously wrong in the undisturbed XSW case (see also Fig. 4). The quantified dose for the $50 \mathrm{keV}$ sample is in good agreement with the nominal value when taking the implantation into account in the XSW calculation.

It should be noted that both techniques are not as sensitive as, e.g., SIMS to channeling or similar effects in the implantation process which result in a deviation from the Gaussian-like behavior of the depth profiles, mainly for the tail of the distribution which extends into the bulk. Indeed, if these effects are not very pronounced, the angle dependent fluorescence signal is dominated by the fraction of the implantation profile which is distributed normally. However, the high sensitivity of the presented grazing XRF techniques to the surface-near regions can be used in combination with other depth profiling techniques, for example SIMS. Such a complementary approach takes advantage of the fact that SIMS is sensitive to possible deviations from a Gaussian-like behavior in the deeper parts of the studied sample, whereas GIXRF and GEXRF can be used to correct for transient region effects which render the SIMS analysis unreliable for the first nanometers. ${ }^{40}$ This approach would also allow the characterization of technologically more relevant, thermally treated implantations for which the depth distributions are not easily parametrizable due to the lack of models and the presented fitting approach for the grazing XRF techniques needs to be adapted.

Table 1 Determined total retained doses by the reference-free GIXRF technique in comparison to GEXRF transfer values (see text). The nominal implanted dose was $10^{16} \mathrm{~cm}^{-2}$ for all samples. The thickness of the top oxide layer on each sample was determined by GIXRF. For the $10 \mathrm{keV}$ sample the results are identical because, in GEXRF, the GIXRF value for this sample is used as a reference to quantify the other samples

\begin{tabular}{lllrl}
\hline & \multicolumn{2}{l}{ Determined dose } & & \\
\cline { 2 - 4 } Implant energy, keV & GIXRF, $10^{15} \mathrm{~cm}^{-2}$ & Improved GIXRF, $10^{15} \mathrm{~cm}^{-2}$ & GEXRF, $10^{15} \mathrm{~cm}^{-2}$ & Oxide thickness, $\mathrm{nm}$ \\
\hline 1 & $5.9 \pm 0.6$ & $6.0 \pm 0.6$ & $8.5 \pm 1.2$ & 4.8 \\
5 & $7.2 \pm 0.7$ & - & $10.4 \pm 1.5$ & 2.8 \\
10 & $9.7 \pm 1.0$ & - & $9.7 \pm 1.0$ & 2.7 \\
15 & $9.6 \pm 1.0$ & $9.3 \pm 0.9$ & $9.9 \pm 1.4$ & 2.7 \\
50 & $6.5 \pm 0.7$ & $9.7 \pm 1.0$ & $9.9 \pm 1.4$ & 1.8 \\
\hline
\end{tabular}




\section{Conclusions}

Both geometries, grazing incidence and grazing emission, allow significant enhancement of the X-ray fluorescence from the surface relative to the bulk by reducing the bulk contribution to the measurement and thus the study of the sample near-surface region with a high sensitivity. Because of the dopant element being just one atomic number smaller than the bulk element, selecting an appropriate primary beam energy was of prime importance in both setups to separate the Si RRS signal, the elastic scattering and the $\mathrm{Al}-\mathrm{K} \alpha$ line for which the background conditions were thus optimized by reducing (GIXRF) or eliminating (GEXRF) any noise source. By evaluating the angular dependence of the X-ray fluorescence intensity on the grazing incidence or emission angles in the vicinity of the respective critical angle, the depth distribution of the emitting sources, i.e., the dopant atoms emitting the fluorescence radiation, can be deduced. Indeed the accessed depth region, spanning from the surface into the sample, can be tuned with the grazing angle. This allows depth-dependent studies of the sample composition. These depth-profiling capabilities of the near surface region were demonstrated for different Al-implanted samples of which the Al concentration distributions could be retrieved. For GIXRF the fitting algorithm had to be adapted to take into account the high dopant concentration and its consequences, e.g., on the XSW field. A comparison with theoretically expected distributions showed a reasonable agreement. For the samples with the highest implantation energies complementary measurements with SIMS were realized. They confirmed the retrieved depth profiles by means of GIXRF and GEXRF as well as the theoretical TRIM predictions for the sample with the highest implantation energy whereas for the second sample, the depth region close to the surface presented ambiguous results. GIXRF and GEXRF confirm thus their potential for quantitative characterization of narrow depth distributions close to the sample interface, a sample configuration where other depth-profiling techniques can struggle.

\section{Acknowledgements}

Parts of this work were supported by the European Commission - Research Infrastructure Action under the FP6 "European Integrated Activity of Excellence and Networking for Nano and Micro-Electronics Analysis" - Project number 026134(RI3) ANNA. The financial support of the Swiss National Science Foundation and the ESRF is also acknowledged. The authors would like to thank P. Helm of RTG Mikroanalyse GmbH for the SIMS measurements.

\section{References}

1 W. Vandervoorst, T. Janssens, R. Loo, M. Caymax, I. Peytier, R. Lindsay, J. Frühauf, A. Bergmaier and G. Dollinger, Appl. Surf. Sci., 2003, 203-204, 371.

2 K. Wittmaack, Surf. Interface Anal., 1998, 26, 290

3 K. L. Yeo, A. T. S. Wee, A. See, R. Liu and C. M. Ng, Appl. Surf. Sci., 2003, 203-204, 335.

4 T. H. Büyüklimanli, C. W. Magee, J. W. Marino and S. R. Walther, J. Vac. Sci. Technol., B: Microelectron. Nanometer Struct-Process., Meas., Phenom., 2006, 24, 408.

5 A. Iida, K. Sakurai, A. Yoshinaga and Y. Goshi, Nucl. Instrum. Methods Phys. Res., Sect. A, 1986, 246, 736.
6 G. Pepponi, C. Streli, P. Wobrauschek, N. Zoeger, K. Luening, P. Pianetta, D. Giubertoni, M. Barozzi and M. Bersani, Spectrochim. Acta, Part B, 2004, 59, 1243.

7 L. Pei, G. Duscher, C. Steen, P. Pichler, H. Ryssel, E. Napolitani, D. De Salvador, A. M. Piro, A. Terrasi, F. Severac, F. Cristiano, K. Ravichandran, N. Gupta and W. Windl, J. Appl. Phys., 2008, 104, 043507.

8 D. Ingerle, F. Meirer, N. Zoeger, G. Pepponi, D. Giubertoni, G. Steinhauser, P. Wobrauschek and C. Streli, Spectrochim. Acta, Part B, 2010, 65, 429.

9 T. W. Barbee Jr and W. K. Warburton, Mater. Lett., 1984, 3, 17.

10 M. Lommel, F. Reinhardt, P. Hönicke, M. Kolbe, M. Müller, B. Beckhoff and B. O. Kolbesen, ECS Trans., 2009, 25, 433.

11 C. Weiss, J. Knoth, H. Schwenke, H. Geisler, J. Lerche, R. Schulz and H. J. Ullrich, Microchim. Acta, 2000, 133, 65.

12 B. Beckhoff, J. Anal. At. Spectrom., 2008, 23, 845.

13 P. Hönicke, B. Beckhoff, M. Kolbe, D. Giubertoni, J. A. van den Berg and G. Pepponi, Anal. Bioanal. Chem., 2010, 396(8), 2825.

14 Y. Kayser, D. Banaś, W. Cao, J.-Cl. Dousse, J. Hoszowska, P. Jagodziński, M. Kavčič, A. Kubala-Kukuś, S. Nowak, M. Pajek and J. Szlachetko, Spectrochim. Acta, Part B, 2010, 65, 445.

15 Y. Kayser, D. Banaś, W. Cao, J.-Cl. Dousse, J. Hoszowska, P. Jagodziński, M. Kavčič, A. Kubala-Kukuś, S. Nowak, M. Pajek and J. Szlachetko, X-Ray Spectrom., 2012, 41, 98.

16 P. de Bokx, C. Kok, A. Bailleul, G. Wiener and H. Urbach, Spectrochim. Acta, Part B, 1997, 52, 829.

17 M. L. Monaghan, T. Nigam, M. Houssa, S. D. Gendt, H. P. Urbach and P. K. de Bokx, Thin Solid Films, 2000, 359, 197.

18 Z. Spolnik, J. Zhang, K. Wagatsuma and K. Tsuji, Anal. Chim. Acta, 2002, 455, 245.

19 J. Hoszowska, J. C. Dousse, J. Kern and C. Rhême, Nucl. Instrum. Methods Phys. Res., Sect. A, 1996, 376, 129.

20 R. S. Becker, J. A. Golovchenko and J. R. Patel, Phys. Rev. Lett., 1983, 50, 153 .

21 H. J. Sánchez, Spectrochim. Acta, Part B, 2001, 56, 2027.

22 H. J. Sánchez, X-Ray Spectrom., 1999, 28, 51.

23 F. Meirer, G. Pepponi, C. Streli, P. Wobrauschek and N. Zoeger, J. Appl. Phys., 2009, 105, 074906.

24 A. Kubala-Kukuś, D. Banás, W. Cao, J.-Cl. Dousse, J. Hoszowska, Y. Kayser, M. Pajek, M. Salomé, J. Susini, J. Szlachetko and M. Szlachetko, Phys. Rev. B: Condens. Matter Mater. Phys., 2009, 80, 113305.

25 B. Beckhoff, R. Fliegauf, M. Kolbe, M. Müller, J. Weser and G. Ulm, Anal. Chem., 2007, 79, 7873.

26 G. H. Vineyard, Phys. Rev. B: Condens. Matter Mater. Phys., 1982, 26, 4146.

27 T. Noma, K. Takada and A. Iida, X-Ray Spectrom., 1999, 28, 433.

28 B. Pollakowski, B. Beckhoff, F. Reinhardt, S. Braun and P. Gawlitza, Phys. Rev. B: Condens. Matter Mater. Phys., 2008, 77, 235408.

29 K. Shinoda, S. Sato, S. Suzuki, T. Uruga, H. Tanida, H. Toyokawa, Y. Terada and Y. Takagaki, J. Surf. Anal., 2009, 15, 295.

30 J. F. Ziegler, M. D. Ziegler and J. P. Biersack, Nucl. Instrum. Methods Phys. Res., Sect. B, 2010, 268(11-12), 1818.

31 F. Senf, U. Flechsig, F. Eggenstein, W. Gudat, R. Klein, H. Rabus and G. Ulm, J. Synchrotron Radiat., 1998, 5, 780.

32 B. Beckhoff and G. Ulm, Adv. X-Ray Anal., 2001, 44, 349.

33 M. Müller, B. Beckhoff, G. Ulm and B. Kanngießer, Phys. Rev. A. At., Mol., Opt. Phys., 2006, 74, 012702.

34 D. Windt, Comput. Phys., 1998, 12, 360.

35 J. Szlachetko, J.-Cl. Dousse, J. Hoszowska, M. Pajek, R. Barrett, M. Berset, K. Fennane, A. Kubala-Kukus and M. Szlachetko, Phys. Rev. Lett., 2006, 97, 073001.

36 H. P. Urbach and P. K. de Bokx, Phys. Rev. B: Condens. Matter Mater. Phys., 2001, 63, 085408.

37 Z. M. Spolnik, M. Claes, R. E. Van Grieken, P. K. de Bokx and H. P. Urbach, Spectrochim. Acta, 1999, 54, 1525.

38 B. L. Henke, E. M. Gullikson and J. C. Davis, At. Data Nucl. Data Tables, 1993, 54, 181.

39 R. Klockenkämper, Total-Reflection X-ray Fluorescence Analysis, Wiley \& Sons, 1996.

40 G. Pepponi, D. Giubertoni, M. Bersani, F. Meirer, D. Ingerle, G. Steinhauser, C. Streli, P. Hönicke and B. Beckhoff, J. Vac. Sci. Technol., B: Microelectron. NanometerStruct.-Process., Meas., Phenom., 2010, 28, C1C59. 